\title{
Patients with progressive pseudorheumatoid dysplasia: From clinical diagnosis to molecular studies
}

\author{
JUN YE, HUI-WEN ZHANG, WEN-JUAN QIU, LIAN-SHU HAN, \\ YA-FEN ZHANG, ZHU-WEN GONG and XUE-FAN GU
}

\begin{abstract}
Department of Pediatric Endocrinology and Genetic Metabolism, Shanghai Institute for Pediatric Research, Xinhua Hospital, Shanghai Jiao Tong University School of Medicine, Shanghai, P.R. China
\end{abstract}

Received May 18, 2011; Accepted September 14, 2011

DOI: $10.3892 / \mathrm{mmr} .2011 .619$

\begin{abstract}
Progressive pseudorheumatoid dysplasia (PPD) is a rare inherited autosomal recessive disease for which no prevalent data have been reported in China. We aimed to identify PPD based on clinical manifestations and imaging analysis of the bony skeleton and then to investigate gene mutations of Wnt1-inducible signaling pathway protein 3 (WISP3) in Chinese patients with PPD. Seven patients (aged 9-49 years) from six unrelated Chinese families all presented with a waddling gait, progressive swelling and restricted joint movements, and all were diagnosed as having PPD according to clinical signs and symptoms, as well as radiographic imaging. The radiographic imaging revealed no erosive arthropathy, but showed platyspondyly, irregular or wedged/ovoid anterior end-plates of the vertebral bodies, coxa vara and widened epiphyses or metaphyses including the femoral head and the metacarpophalangeal and interphalangeal joints. Normal laboratory values were found for the erythrocyte sedimentation rate, C-reactive protein and rheumatoid factors in all patients. Molecular studies revealed that five patients carried c.624_625insA/c.729_735delGAGAAAA, c.624_625insA/ c.866_867insA, c.866_867 insA/c.866_867insA, Q46X/ $\mathrm{C} 114 \mathrm{~W}$ and $\mathrm{C} 223 \mathrm{G} / \mathrm{C} 114 \mathrm{~W}$ mutations, respectively. In conclusion, our findings suggest that in order to avoid misdiagnosis, physicians should carefully examine the entire skeleton, including the spine, in addition to the skeletal extremities. Mutation analysis of the WISP3 gene is useful for confirming the clinical and radiographic diagnosis of PPD.
\end{abstract}

\section{Introduction}

Progressive pseudorheumatoid dysplasia (PPD) (OMIM 208230) is an inherited autosomal recessive skeletal dysplasia with

Correspondence to: Dr Jun Ye, Shanghai Institute for Pediatric Research, Xin Hua Hospital, Shanghai Jiao Tong University School of Medicine, 1665 Kong Jiang Road, Shanghai 200092, P.R. China

E-mail: yejun2314@yahoo.com.cn

Key words: progressive pseudorheumatoid dysplasia, platyspondyly, WISP3, mutation, Chinese radiographic changes in the spine similar to spondyloepiphyseal dysplasia tarda, progressive arthropathy (SEDT-PA) and with a clinical resemblance to juvenile idiopathic arthritis (JIA). In the 1997 revision of the International Nomenclature and Classification of Osteochondrodysplasias, PPD is listed as an autosomal recessive disorder in group 10, i.e. 'other spondyloepi-(meta)-physeal [SE(M)D] dysplasias' (1). The incidence of PPD has been estimated at 1 individual per million in the UK (2), but it is likely to be higher in the Mediterranean, Middle East and Gulf states $(3,4)$. No prevalent data on the Chinese population have been reported as yet.

PPD is a rare disease which has been attributed to mutations in the gene encoding the Wnt1-inducible signaling pathway protein 3 (WISP3) (OMIM 603400). The WISP3 gene is located on chromosome $6 \mathrm{q} 22$ and is composed of five coding exons, which encode a 354-amino acid protein (5). The articular chondrocytes (ACs) in PPD are characterized by increased proliferative activity and decreased apoptosis, with continuous degeneration and loss of articular cartilage being the main pathological consequences of the disease (6). Patients with PPD suffer from a progressive, non-inflammatory chondropathy affecting primarily the articular cartilage, with characteristic skeletal abnormalities, notably in the spine and joints. It is often initially misdiagnosed as an inflammatory rheumatic disease, such as JIA $(7,8)$ or mucopolysaccharidosis. Therefore, it is crucial to carry out an early differential diagnosis. The objective of this study was to report on seven patients from six Chinese families identified as having PPD from clinical manifestations and the imaging analysis of the bony skeleton. A further aim was to explore the gene mutation spectrum of WISP3 in the Chinese patients with PPD to confirm the clinical diagnosis at the molecular level.

\section{Materials and methods}

Patient histories. Seven patients (six males and one female) from six Chinese families were included in this study over a period of two years and nine months (March 2008 to December 2010). Patient 7 is the fourth younger sister of patient 6. All of these patients were delivered at a normal length and weight following a full-term, uneventful preg- 
nancy. The age of disease onset in the seven patients was 5-13 years, when they presented with limb muscle weakness or pain in the hip after walking for longer distance or climbing stairs, and/or a waddling gait. Their proximal and distal interphalangeal joints were swollen at the age of 6-15 years (Fig. 1), and the joint swelling and/or pain gradually progressed to the large joints (Fig. 2). The skin was not red or warm over the affected joints, but developed stiffness and flexion contracture, resulting in motion limitation of the joints, such as being unable to bend from the waist, turn the head, or squat in six patients, and unable to walk unaided in two patients at the age of 9-17 years. The detailed symptoms and signs of these patients are shown in Table I. All patients have normal intelligence. Six of these patients were suspected as having rheumatoid polyarthritis in a local hospital, but nonsteroidal anti-inflammatory drugs were of no clinical benefit. These patients visited our clinic at the age of 9-49 years for short stature (their current height is near or less than the 3rd percentile) and motion limitation of the joints. The other family members of the five patients (P1-P5) are all healthy. The patient P6 and his youngest sister (P7) from one family are affected, but his parents, younger brother and younger sister are all healthy.

Clinical diagnosis. Laboratory evaluation included routine blood, liver and renal function, serum calcium phosphorus and alkaline phosphatase levels, as well as the erythrocyte sedimentation rate, $\mathrm{C}$ reactive protein, rheumatoid factors, antistreptolysin $\mathrm{O}$ and antinuclear antibodies to exclude connective tissue disease. We also analyzed the urinary excretion of glycosaminoglycans by quantitative dimethylmethylene blue test, or enzyme assay for mucopolysaccharidosis type I, II, IV and VI, to rule out mucopolysaccharidosis. Radiological investigations (X-rays of the spine, hip, knee and hand) and magnetic resonance imaging of the spine were performed to make a radiological diagnosis of PPD.

Molecular studies. Written informed consent was obtained from the patients' parents prior to blood sampling and DNA analysis. This study was approved by the Ethics Committee of the Shanghai Jiaotong University School of Medicine, China. DNA was extracted from the peripheral white blood cells of patients and their parents using conventional methods. All 5 exons and the exon-intron boundaries of the WISP3 gene were amplified by polymerase chain reaction (PCR) using five pairs of primers (Table II). Genomic DNA $(2 \mu 1)$ was used as a template in a total reaction volume of $50 \mu 1(5 \mu 1$ 10X buffer plus $\mathrm{Mg}^{2+}, 5 \mu \mathrm{l} 2.5 \mathrm{mM}$ dNTP mixture, $1.5 \mu \mathrm{l}$ each primer $20 \mu \mathrm{M}, 2.5 \mu \mathrm{l}$ Takara Taq and $\mathrm{dH}_{2} \mathrm{O}$ up to $50 \mu \mathrm{l}$ ). PCR was performed at an initial denaturation time of $95^{\circ} \mathrm{C}$ for $5 \mathrm{~min}$, followed by 34 cycles of $94^{\circ} \mathrm{C}$ for $45 \mathrm{sec}, 62^{\circ} \mathrm{C}$ for $40 \mathrm{sec}$ and $72^{\circ} \mathrm{C}$ for $1 \mathrm{~min}$, and a final extension interval at $72^{\circ} \mathrm{C}$ for $10 \mathrm{~min}$. Direct DNA sequencing was performed in both directions by a commercial sequencing service (Shanghai GeneCore Biotechnologies, China). The reference WISP3 gene sequence used was GenBank NG_011748.1. The non-previously described variations were tested by only mutation screening in 50 normal controls (40 males, 10 females) who were disease-unrelated Han Chinese children aged 5-20 years.
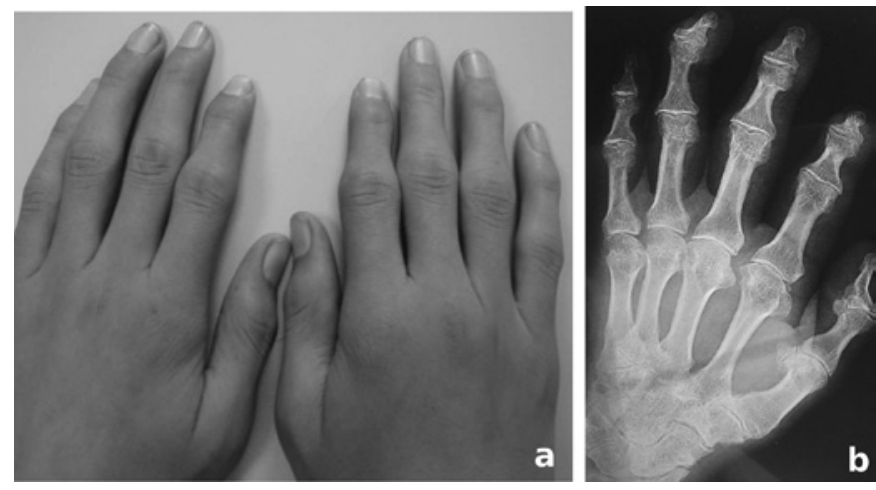

Figure 1. Images of a representative patient. (a) Hands with enlargement of the interphalangeal joints, and (b) hand radiograph showing enlargement of the metaphyses and epiphysis at the metacarpals and phalanges, with loss of joint space.
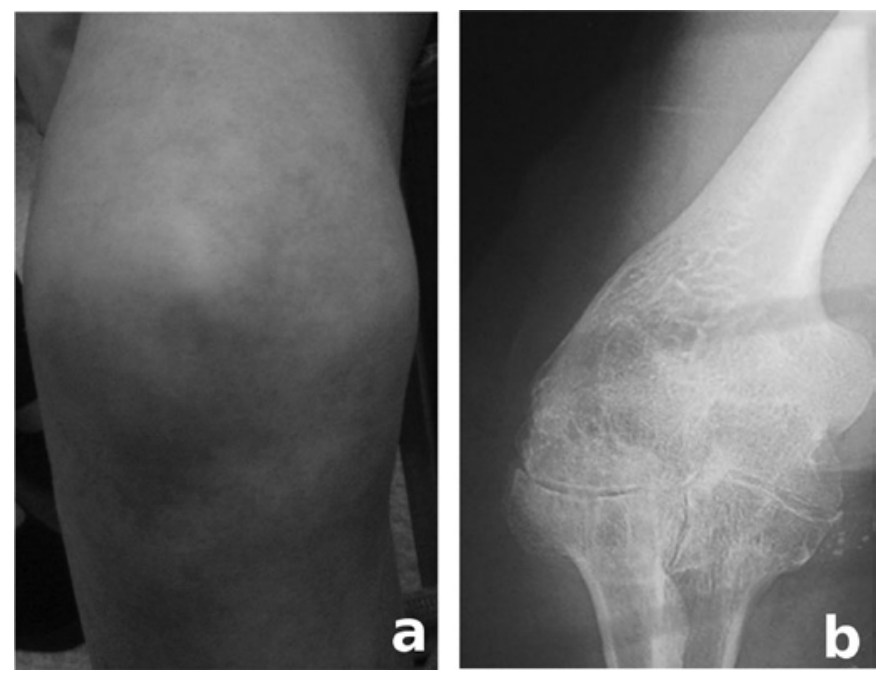

Figure 2. Images of a representative patient. (a) Enlarged knee joints and (b) knee radiograph showing widened metaphyses at the distal femur and proximal tibia and loss of joint space of the knee with periarticular osteoporosis.

\section{Results}

Laboratory findings. Seven patients in this study were initially suspected as having an inflammatory rheumatic disease or mucopolysaccharidosis. The erythrocyte sedimentation rate, $\mathrm{C}$ reactive protein, rheumatoid factor, antinuclear antibody and quantitative dimethylmethylene blue tests were negative in all patients. The enzyme activities of mucopolysaccharidosis type I, II, IV and VI performed in patient 2 were within the normal range. None of the above laboratory findings gave any evidence for a diagnosis of connective tissue disease or mucopolysaccharidosis.

Radiological findings. Radiological examination in the seven patients did not reveal any erosive arthropathy, but did show universal platyspondyly, irregular (bird mouth-like) or wedged/ovoid anterior end-plate of the vertebral bodies, particularly in the thoracolumbar region (Fig. 3). Five patients (P1, P4-P7) had loss of joint space in the hip joints, irregularities or cystic changes of the articular surface of the femoral head and acetabulum, and a shortened femoral neck (Fig. 4). 
Table I. Symptoms and signs of the seven patients presenting with PPD.

\begin{tabular}{|c|c|c|c|c|c|c|c|}
\hline & \multicolumn{7}{|c|}{ Patient ID } \\
\hline & P1 & $\mathrm{P} 2$ & $\mathrm{P} 3$ & P4 & P5 & P6 & P7 \\
\hline Gender & $\mathrm{M}$ & M & $\mathrm{M}$ & $\mathrm{M}$ & $\mathrm{M}$ & $\mathrm{M}$ & $\mathrm{F}$ \\
\hline Age of onset (years) & 7 & 8 & 5 & 6 & 7 & 14 & 13 \\
\hline Muscle weakness & + & + & + & + & + & + & + \\
\hline Waddling gait & + & + & + & + & + & + & + \\
\hline Genu varum & - & - & - & - & + & - & - \\
\hline Swollen interphalangeal joints & + & + & + & + & + & + & + \\
\hline $\begin{array}{l}\text { Larger joint (such as the wrists, elbow } \\
\text { and knee) swelling }\end{array}$ & + & + & - & + & - & + & + \\
\hline Pain in joints & + & + & + & + & - & + & + \\
\hline Stiffness, flexion contracture & + & + & + & + & - & + & + \\
\hline Motion limitation of the joints or spine & + & + & + & + & - & + & + \\
\hline Unable to walk unaided & + & - & - & - & - & + & - \\
\hline Short stature & + & + & + & + & + & + & + \\
\hline Short neck & + & - & + & + & - & + & + \\
\hline Scoliosis & + & - & - & + & + & + & - \\
\hline Family history & - & - & - & - & - & + & + \\
\hline
\end{tabular}

Table II. Primers for the PCR amplification of the WISP3 gene.

\begin{tabular}{|c|c|c|c|}
\hline Amplicon & Primer sequence & $\begin{array}{c}\text { Annealing } \\
\text { temperature }{ }^{\circ} \mathrm{C}\end{array}$ & Amplicon size (bp) \\
\hline Exon 1 & $\begin{array}{l}\text { 5'ACGGCGACNTCTCTACC 3' } \\
\text { 5'CTCTTCCTCCATCTTCGTTT 3' }\end{array}$ & 60 & 189 \\
\hline Exon 2 & $\begin{array}{l}\text { 5'ACCTGTTTGGGGGAAATCTT 3' } \\
\text { 5'CCAAGCTAACAATTGCAGGA 3' }\end{array}$ & 60 & 459 \\
\hline Exon 3 & 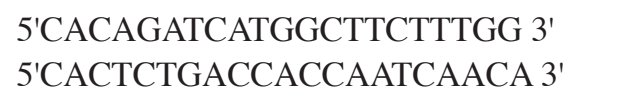 & 60 & 399 \\
\hline Exon 4 & $\begin{array}{l}\text { 5'CCATCGGCTTCTTTTATTCTG 3' } \\
\text { 5'CACACACATGCTTACTTTTCAGG 3' }\end{array}$ & 62 & 396 \\
\hline Exon 5 & 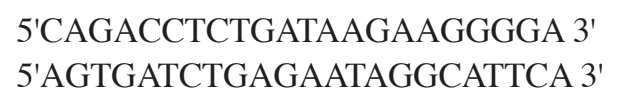 & 62 & 575 \\
\hline
\end{tabular}

Five patients (P1, P2, P4, P6, P7) had scoliosis, kyphosis and coxa vara, and widened epiphyses or metaphyses, including the capital femoral, distal femur and proximal tibia. Three patients (P1, P6, P7) had loss of joint space of the knee with periarticular osteoporosis (Fig. 2b). Six patients (P1-P4, P6, P7) had enlarged metacarpophalangeal and interphalangeal joints with joint space narrowing (Fig. 1b). These radiological findings, combined with the clinical symptoms and laboratory results, markedly suggested the diagnosis of PPD for the seven patients.

Molecular findings. We sequenced all five coding exons of WISP3 for seven patients with PPD and their parents, and also the younger sister of patient 4 . The healthy second younger sister and the younger brother of patient 6 received the gene mutation analysis. Seven different mutations of the WISP3 gene were identified with a total detection rate of $91.67 \%$ (11/12). These mutations included c.866_867insA (27.27\%), c.624_625insA (18.18\%), c.7210T>G(p. C114W) (18.18\%), c.729_735delGAGAAAA, c.7004C $>$ T (p.Q46X), c.14108T>G(p.C223G) and c.208_209insA. We identified compound heterozygous mutations (c.624_625insA/ c.729_735delGAGAAAA) in patient 1, compound heterozygous mutations (c.624_625insA/c.866_867insA) in patient 2, a homozygous mutation (c.866_867insA/c.866_867insA) in patient 3, compound heterozygous mutations (Q46X/ $\mathrm{C} 114 \mathrm{~W}$ ) in patient 4 and compound heterozygous mutations $(\mathrm{C} 223 \mathrm{G} / \mathrm{C} 114 \mathrm{~W})$ in patient 5 . Only one heterozygous muta- 

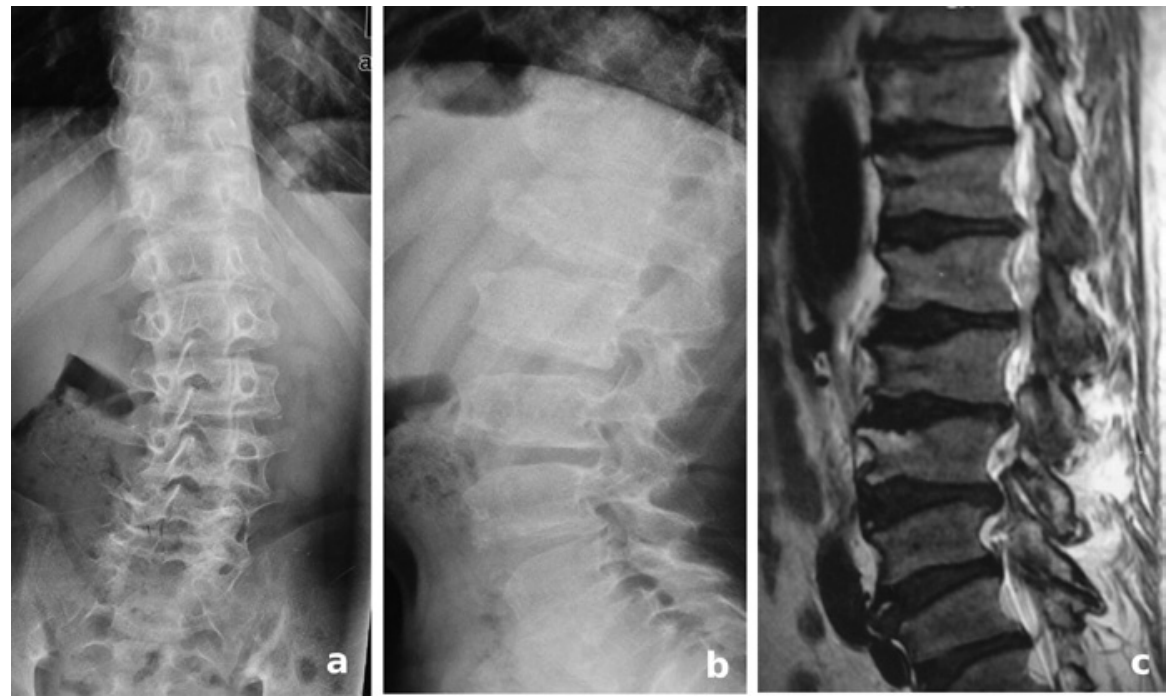

Figure 3. Radiograph of the thoracolumbar spine in a representative patient. (A) Scoliosis, (B) lateral X-rays and (C) T1-weighted magnetic resonance image showing platyspondyly, irregular anterior end-plate of the vertebral bodies (bird mouth-like).

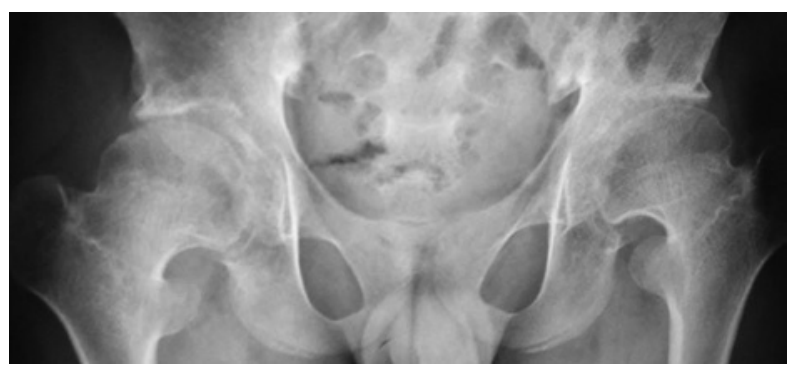

Figure 4. X-ray film of the pelvis showing loss of the hip joint space, coxa vara, irregularities or cystic changes of the articular surface of the femoral head and acetabulum, short and wide femoral neck, and large capital femoral epiphyses.

tion (c.208_209insA) was identified in patients 6 and 7. The mutations carried by patients $1-5$ were also identified in both of their parents, whereas the younger sister of patient 4 carried the $\mathrm{C} 114 \mathrm{~W}$ mutation only. The mutation carried by patient 6 and patient 7 came from their father. The healthy second younger sister and younger brother of patient 6 did not carry the c.208_209insA mutation. The maps of gene mutation in the seven patients are shown in Fig. 5. Three insertion mutations (c.866_867insA, c.624_625insA and c.208_209insA) and one deletion mutation (c.729_735delGAGAAAA) resulted in a frameshift. One nonsense mutation (G46X), which is a C to $\mathrm{T}$ transition at c.7004 in exon 2, changed the codon Gln to Ter and resulted in a subsequent change to a stop codon and a truncation at p.46. One missense mutation $\mathrm{C} 114 \mathrm{~W}$, which is a $\mathrm{T}$ to $\mathrm{G}$ transition at c.7210 in exon 2, resulted in a Cys to Trp substitution at p.114; and the other missense mutation, C223G, which is a $\mathrm{T}$ to $\mathrm{G}$ transition at c.14108 in exon 4, resulted in a Cys to Gly substitution at p.223. Four of seven types of mutations were located on exon 4 (57.14\%), two were located on exon $2(28.57 \%)$ and one was located on exon $5(14.28 \%)$ of the WISP3 gene. Three mutations, c.624_625insA, c.866_867insA
A

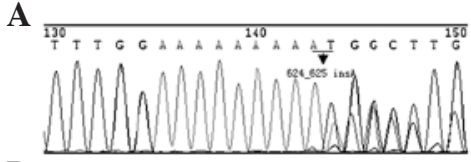

D

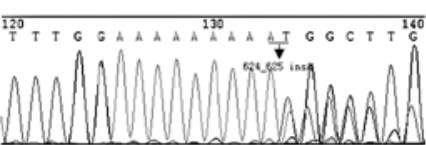

G

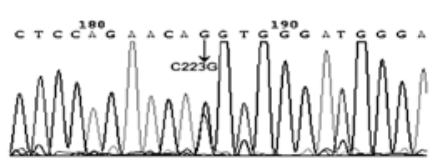

$\mathbf{B}$

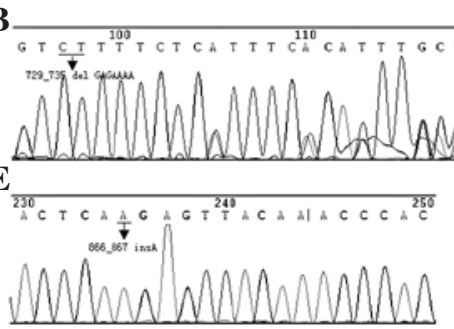

$\mathbf{H}$

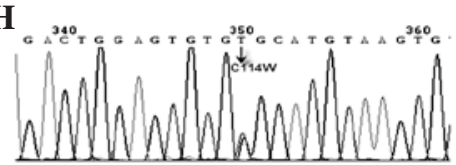

C

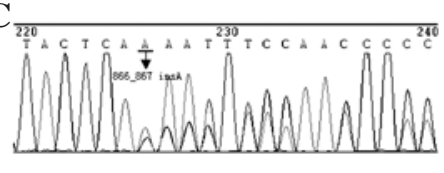

F

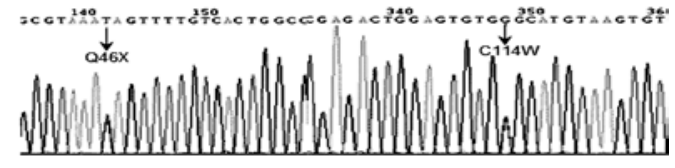

I

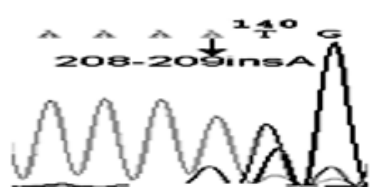

Figure 5. Maps of the WISP3 gene mutations in the seven patients. (A and B) Compound heterozygous mutations, c.624_625insA and c.729_735delGAGAAAA, (reverse map) carried by patient 1. (C and D) The compound heterozygous mutations, c.866_867 ins A and c.624_625insA, carried by patient 2.(E) Homozygous mutation, c.866_867insA, carried by patient 3. (F) The compound heterozygous mutations, Q46X and C114W, carried by patient 4. (G and H) The compound heterozygous mutations, $\mathrm{C} 223 \mathrm{G}$ and C114W, carried by patient 5. (I) A heterozygous mutation, 208-209insA, carried by patient 6 and patient 7 . 
and c.729_735delGAGAAAA, have been reported by our group in a Chinese journal (9). The other four mutations have not been previously reported and were not identified in 100 control chromosomes.

\section{Discussion}

PPD is a rare hereditary polyarthropathy involving the small and large joints. In China, with the gradual development of the field of genetic metabolic diseases over the last decade, a number of diseases such as PPD are now capable of being diagnosed at an early stage. However, as the incidence of PPD across the world is very low, it has not been easy to find a number of patients with PPD within a certain period of time. To date, only a small number of Chinese patients with PPD have been reported $(6,10)$. There is no prevalent data of PPD in China at present.

It has been reported that in PPD, the ACs, which are the predominant cell components of articular cartilage, proliferate significantly faster than normal cells, and have a lower rate of apoptosis compared to normal controls. These findings may account for the metaphyseal enlargement observed in PPD radiographs, which corresponds clinically to the joint swelling. The continuous degeneration and loss of articular cartilage are the main pathological changes of PPD (6). In contrast to the majority of genetic skeletal dysplasias, prenatal skeletal growth and morphogenesis appear undisturbed in PPD, and the affected individuals are asymptomatic during the first few years of life (5). In our study, 85.71\% (6/7) of our patients were male; thus the incidence appears to be higher in males. The onset of the disease in our patients was between 5 and 13 years old, with presenting symptoms of muscle weakness, waddling gait, interphalangeal joints which were swollen and then gradually developed deformities, stiffness, swelling and motion-range limitation of symmetric multiple large joints as they aged in the majority of the patients. These patients were ultimately diagnosed as having PPD based on clinical and radiographic criteria (11). Two patients (P1, P6) progressed to be unable to stand and walk unaided. Such functional impairment in PPD may develop as a result of a combination of joint abnormalities together with muscle weakness and wasting. The prognosis may actually be very poor, including the possibility of becoming wheelchair-bound at an early age, although life expectancy is not likely to be diminished $(8,11)$. On the other hand, the current height of each patient is near or less than the 3rd percentile of the mean height of normal children. The reduction in height is explained by the progressive contraction in the hips as well as the spinal deformity (12). Patients with PPD typically receive no significant benefit from treatment with nonsteroidal anti-inflammatory drugs (NSAIDs). Kaya et al (13) reported that the pain was controlled with NSAIDs, electrotherapy [transcutaneous electrical nerve stimulation (TENS) and diadynamic current] and superficial heat (paraffin bath and hot pack), and strength exercises were useful to maintain and/ or increase muscle strength and range of motion.

Our patients with PPD were initially misdiagnosed as having an inflammatory rheumatic disease, such as JIA and mucopolysaccharidosis. PPD should be considered by clinical radiologists and pediatricians in conducting the differential diagnosis for these diseases, particularly when laboratory support is lacking. It is essential to carefully examine the entire skeleton, including the spine, and not to concentrate exclusively on the extremities of the skeleton in order to prevent delayed diagnosis and to begin rehabilitation as early as possible. PPD should be distinguished from certain other diseases of the bone and joints $(8,11,14)$. These diseases include: i) JIA with symmetric or asymmetric joint involvement with bony erosions and without spondyloepiphyseal dysplasia, accompanied by iridocyclitis, anemia, leukocytosis, altered erythrocyte sedimentation rate and $\mathrm{C}$ reactive protein elevation, positive antinuclear antibodies and rheumatoid factor; ii) Stickler syndrome, a dominantly inherited disease characterized by progressive myopia, hearing obstruction and polyarthropathy; iii) spondyloepiphyseal dysplasia tarda, which is mainly an X-linked disorder and develops at an older age, with shortness, vertebral flattening and osteoarthritis in the large joints, particularly the hips, as a common complication, and the relatively unaffected peripheral skeleton including the interphalangeal joints; and iv) mucopolysaccharidosis, with characteristic features including platyspondyly, bullet or wedged anterior end-plates of vertebral bodies and banderole ribs, but with small joints of the hand and larger joints such as the knee appearing to be normal. The quantitative dimethylmethylene blue test and the determination of enzymatic activity allow confirmation of the diagnosis.

The WISP3 gene regulates the synthesis of chondrocyte collagen type II and saccharan, and the latter two are the major components of cartilaginous tissue. Therefore, any depletion or dysfunction of WISP3 may result in a loss of WISP3 protein function, which apparently interferes with normal postnatal growth and cartilage homeostasis, leading to joint degeneration $(5,8,15,16)$. The mutation analysis of the WISP3 gene may confirm the original diagnosis of PPD based on clinical manifestations and imaging analysis. Mutation analysis may also assist pediatricians and radiologists in recognizing certain phenotypes at an early stage of PPD. To date, over 20 mutations in the WISP3 gene have been identified in PPD families including exon-deletion/insertion, nonsense and missense mutations. In this study, we describe the molecular analysis of WISP3 performed in seven patients from six families with PPD. We identified seven types of mutations including three mutations reported previously by our group (9). All these mutations in the WISP3 gene are different from the mutations in other Chinese patients with PPD reported by Zhou et al and Yue et al $(6,10)$ and have not been previously reported by investigators in other countries around the world. Each novel mutation carried by our patients was also identified in the father or mother. The two novel missense mutations were not found in 100 control chromosomes, strongly suggesting that they are not a common polymorphism. It therefore appears that these novel variations carried by our patients are disease-causing mutations, although a gene expression study has not yet been performed. On the other hand, the majority of the mutations we reported here are located on exon 4, and the others are located on exon 2 and exon 5 of the WISP3 gene, while the mutations identified in another two Chinese families reported previously are located on exon 3 (10), indicating that the mutations of the WISP3 gene in Chinese PPD are located in the range of exon 2 to exon 5. Although the mutations are located on different exons, the 
patients had very similar phenotypes, suggesting that the mutations in different domains of WISP3 are associated with similar clinical features. The correlation between the phenotype and genotype of PPD remains to be clarified at the molecular level and requires further analysis in future research.

\section{Acknowledgements}

The authors are grateful to the patients' families for their collaboration. This work was supported by the National Key Technology R\&D Program (no. 2006BAI05A05, no. 2006BAI05A07), the '863' Program (no. 2007AA02Z447) and the Shanghai Jiao Tong University School of Medicine Foundation Technology (no. 06XJ21024).

\section{References}

1. Lachman RS: International nomenclature and classification of the osteochondrodysplasias. Pediatr Radiol 28: 737-744, 1998.

2. Wynne-Davies R, Hall C and Ansell BM: Spondylo-epiphysial dysplasia tarda with progressive arthropathy. A 'new' disorder of autosomal recessive inheritance. J Bone Joint Surg Br 64 442-445, 1982

3. El-Shanti H, Murray JC, Semina EV, Beutow KH, Scherpbier T and Al-Alami J: Assignment of gene responsible for progressive pseudorheumatoid dysplasia to chromosome 6 and examination of COL10A1 as a candidate gene. Eur J Hum Genet 6: 251-256, 1998.

4. Teebi AS and Al Awadi SA: Spondyloepiphyseal dysplasia tarda with progressive arthropathy: A rare disorder frequently diagnosed among Arabs. J Med Genet 23: 189-191, 1986.

5. Hurvitz JR, Suwairi WM, Van Hul W, El-Shanti H, Superti-Furga A, Roudier J, HolderbaumD, Pauli RM, Herd JK, Van Hul EV, Rezai-Delui H, Legius E, Le Merrer M, Al-Alami J, Bahabri SA and Warman ML: Mutations in the CCN gene family member WISP3 cause progressive pseudorheumatoid dysplasia. Nat Genet 23: 94-98, 1999.
6. Zhou HD, Bu YH, Peng YQ, Xiu H, Wang M, Yuan LQ, Jiang Y, Li D, Wei QY, He YL, Xiao T, Ni JD and Liao EY: Cellular and molecular responses in progressive pseudorheumatoid dysplasia articular cartilage associated with compound heterozygous WISP3 gene mutation. J Mol Med 85: 985-996, 2007.

7. Byrne PA and Rajan KT: Spondylo-epiphyseal dysplasia tarda with progressive arthropathy mimicking juvenile chronic arthritis. Br J Rheumatol 37: 233-234, 1998.

8. Ehl S, Uhl M, Berner R, Bonafe L, Superti-Furga A and Kirchhoff A: Clinical, radiographic, and genetic diagnosis of progressive pseudorheumatoid dysplasia in a patient with severe polyarthropathy. Rheumatol Int 24: 53-56, 2004.

9. Ye J, Zhang HW, Wang T, Cao LF, Qui WJ, Han LS, Zhang YF and Gu XF: [Clinical diagnosis and WISP3 gene mutation analysis for progressive pseudorheumatoid dysplasia]. Zhonghua Er Ke Za Zhi 48: 194-198, 2010 (In Chinese).

10. Yue H, Zhang ZL and He JW: Identification of novel mutations in WISP3 gene in two unrelated Chinese families with progressive pseudorheumatoid dysplasia. Bone 44: 547-554, 2009.

11. Bennani L, Amine B, Ichchou L, Lazrak N and HajjajHassouni N: Progressive pseudorheumatoid dysplasia: Three cases in one family. Joint Bone Spine 74: 393-395, 2007.

12. El-Shant HE, Omari HZ and Qubain HI: Progressive pseudorheumatoid dysplasia: report of a family and review. J Med Genet 34: 559-563, 1997.

13. Kaya A, Ozgocmen S, Kiris A and Ciftci I: Clinical and radiological diagnosis of progressive pseudorheumatoid dysplasia in two sisters with severe polyarthropathy. Clin Rheumato 124: $560-564,2005$

14. Shivanand G, Jain V and Lal H: Progressive pseudorheumatoid chondrodysplasia of childhood. Singapore Med J 48: e151-e153, 2007.

15. Delague V, Chouery E, Corbani S, Ghanem I, Aamar S, Fischer J, Levy-Lahad E, Urtizberea JA and Mégarbané A: Molecular study of WISP3 in nine families originating from the Middle-East and presenting with progressive pseudorheumatoid dysplasia: identification of two novel mutations, and description of a founder effect. Am J Med Genet A 138A: 118-126, 2005

16. Nakamura Y, Weidinger G, Liang JO, Aquilina-Beck A, Tamai K, Moon RT and Warman ML: The CCN family member Wisp3, mutant in progressive pseudorheumatoid dysplasia, modulates BMP and Wnt signaling. J Clin Invest 117: 3075-3086, 2007. 